\title{
Transdermal Fentanyl Solution Provides Long-term Analgesia in the Hind-paw Incisional Model of Postoperative Pain in Male Rats
}

\author{
JOHANNE CLEMENSEN*, LENA V. RASMUSSEN* and KLAS S.P. ABELSON \\ Department of Experimental Medicine, Faculty of Health and Medical Sciences, \\ University of Copenhagen, Copenhagen, Denmark
}

\begin{abstract}
Background: A recently developed transdermal fentanyl solution was hypothesized to be effective for noninvasive postoperative analgesia in rats. Materials and Methods: Thirty-nine male Sprague-Dawley rats were treated once with $0.1,0.33$ or $1.0 \mathrm{mg} / \mathrm{kg}$ transdermal fentanyl solution at the skin of the scruff $1 \mathrm{~h}$ prior to subjected to hind-paw surgery, and compared to non-treated animals. All rats were tested for nociceptive response in the electronic von Frey (EVF) test between 1 and $72 \mathrm{~h}$ postoperatively, and assessed daily with regards to facial expression, body weight changes and welfare score. Results: Fentanyl treatment at all doses significantly reduced nociceptive response in the EVF test throughout the $72 \mathrm{~h}$ of experimentation, reduced facial expressions on all days postoperatively, slightly reduced the body weight and improved postoperative welfare parameters. Conclusion: The present study indicates that transdermal fentanyl solution seems to be an effective, non-invasive and long-lasting analgesic regimen in male rats.
\end{abstract}

Laboratory rats are frequently used in various studies in which they are subjected to surgical procedures. This is associated with intra-and postoperative pain and stress (13 ), which should be treated with an appropriate analgesic strategy. The analgesic strategy should ensure sufficient pain relief and optimal recovery of the animal. This means that

This article is freely accessible online.

*These Authors contributed equally to this work.

Correspondence to: Klas S.P. Abelson, Associate Professor, Ph.D., Department of Experimental Medicine, University of Copenhagen, Blegdamsvej 3B, DK-2200, Copenhagen N, Denmark. Tel: +45 35335575, e-mail: klasab@ sund.ku.dk

Key Words: Laboratory rats, pain, analgesia, transdermal, fentanyl. the drug administered should provide antinociception of sufficient efficacy and duration, and that the administration itself should cause as little stress as possible. This makes parenteral administration of drug formulations with short duration of action less suitable, since they call for repeated injections, often several times per day, which is both stressful to the animals and resource-demanding (4). Therefore, novel analgesic strategies have been developed, including oral administration by voluntary intake particularly of buprenorphine mixed in drinking water (5), hydrogels (6-8), nut-paste (9-13), or regular feed pellets (14). These oral strategies are often advantageous compared to injections, since the animals have been shown to be less stressed while still being protected against postoperative pain and stress (9-13). However, the obvious disadvantage with oral voluntary ingestion is the occasional reluctance of animals to ingest the vehicle, which makes the strategy less reliable. In recent years, commercially available buprenorphine in a sustained release formula has been developed, which has enabled a more reliable strategy. This formula is injected subcutaneously by a single injection, after which antinociceptive effects persist demonstrated for several days $(15,16)$. However, the formulations are unavailable in e.g. Europe at present, or not available for research purpose in certain states in the US. Furthermore, a limitation with buprenorphine is its lack of efficacy against more severe pain conditions, which calls for multimodal analgesic strategies or use of stronger opioids, such as fentanyl (17). Fentanyl, in turn, is a very short-acting opioid, which makes parenteral injections of the drug less suitable as an analgesic strategy. However, the use of a transdermal fentanyl formulation (TFS), where the drug is deposited on the skin of the animal, was recently developed for dogs. This strategy has been demonstrated to give analgesic coverage for up to 4 days postoperatively $(18,19)$. If such a strategy were also applicable in laboratory rats, limitations of the postoperative analgesia strategies described above could be overcome. 
The aim of the present study was to investigate and validate possible analgesic effects and positive effects of transdermal fentanyl solution on recovery in male rats in a model of postoperative pain. It was hypothesized that preemptive treatment with the transdermal fentanyl would reduce hyperalgesic response in the electronic von Frey test (EVF), reduce pain-related scores in assessment with the Rat Grimace Scale (RGS), prevent body weight loss, and reduce adverse effects in an assessment of welfare parameters over $72 \mathrm{~h}$ postoperatively.

\section{Materials and Methods}

Ethics statement. All animal experimentation in the present study was reviewed and approved by the Danish Council for Animal Experiments under the Ministry of Environment and Food (license number 2015-15-0201-00644), and by the local animal welfare body at the University of Copenhagen. The animals were housed in a facility fully accredited by the Association for the Assessment and Accreditation of Laboratory Animal Care and were handled by trained personnel in accordance with the Guide for the Care and Use of Laboratory Animals (20).

Animals. In total, 39 male Sprague-Dawley rats (BomTac:SD) from Taconic, Ry, Denmark were used in the study. The animals were approximately 8 weeks old and weighed on average $299 \pm 3 \mathrm{~g}$ [mean \pm standard error of the mean (SEM)] at the start of the study. Only male rats were used in this study. We acknowledge that this is a limitation to the study, since female subjects may be expected to have different baseline pain sensitivities and different responses to analgesic treatments. The reason for omitting females was that we intended to investigate whether the analgesic strategy would be applicable at all. We are, however, intending to follow-up the present study with a study of this strategy, including more parameters and animals of several strains and of both sexes.

Housing. Animals were single-housed from the day of arrival, and throughout the entire study, in order to avoid any cross-contamination of fentanyl due to licking on the treated surface by a cage mate. Animals were housed in individually ventilated macrolone cages (type III, $3822 \times 17 \mathrm{~cm}$ ) from Techniplast, Buguggiate, Italy. Wooden chips (Tapvei Oy, Kortteinen, Finland) were used as bedding material. Bite bricks (Tapvet, Tapvei Oy), Enviro-dri nesting materials (Shepherd Specialty Papers, Quakertown, PA, USA), and cardboard houses (Brogaarden, Gentofte, Denmark) were used for environmental enrichment. A diurnal rhythm was maintained with a 12:12 h light-dark cycle and with artificial light from 06:00 h. Cage temperature was kept at $21-22^{\circ} \mathrm{C}$, relative humidity at $50-10 \%$, and air was exchanged 75 times per hour. Rats were fed ad libitum with Altromin 1319 pellets (Brogaarden), and acidified tap water ( $\mathrm{pH} \mathrm{3.0)}$ was available at all times. The facility follows the guidelines for health monitoring in rodent facilities by the Federation of European Laboratory Science Associations (FELASA) (21). Sentinel rats tested positive for Helicobacter spp. but none of the other pathogens on the FELASA list.

Study design. Animals were randomly allocated to four different groups. Group size was estimated with a power analysis based on the expected effect in the EVF test according to similar studies in our laboratory (unpublished data). Animals without analgesic treatment were expected to respond with $65 \%$ of the preoperative value in the EVF test, with a standard deviation of $25 \%$, which was expected to return to $100 \%$ in fentanyl-treated animals. The alpha level was set to 0.05 and the power to 0.8 , which resulted in an estimated group size of at least eight animals per group.

All groups underwent surgery as described below. One group $(n=12)$ was not treated with any preoperative analgesia, and three groups were treated with transdermal fentanyl at different concentrations $1 \mathrm{~h}$ prior to surgery ( $\mathrm{n}=9$ animals in each group). The postoperative analgesia and recovery of the animals were then assessed with the EVF test, RGS, body weight change and with a welfare assessment protocol, 1-72 $\mathrm{h}$ after surgery, as described in detail below.

All assessments were conducted in a blinded fashion, where the experimenters were unaware of which treatment each animal was subjected to. Surgical procedures and assessments were performed by two experimenters. To avoid any inter-experimenter bias related to the surgical procedures, treatment groups were assigned to both experimenters in a fashion that ensured that all treatment groups were equally distributed and randomized between both experimenters.

Drug administration. The transdermal fentanyl solution used was Recuvyra (Elanco Animal Health, Indianapolis, IN, USA), a clear, colourless to light yellow solution containing $50 \mathrm{mg} / \mathrm{ml}$ fentanyl combined with isopropanol as solvent, and the skin penetration enhancer octyl salicylate (22). The solution was applied $1 \mathrm{~h}$ before surgery to the skin (after hair removal) in the dorsal interscapular region with the use of a micropipette. The solution was applied in volumes of $0.6,2.0$ and $6.0 \mu \mathrm{l}$ respectively, which correspond to approximate doses of $0.1,0.33$ and $1.0 \mathrm{mg} / \mathrm{kg}$ bodyweight. Animals in the control group were handled in the same way as the fentanyltreated animals, but no liquid was applied to the skin.

Surgical procedure. In order to induce a significant postoperative pain condition, a hind-paw incision described previously by Brennan et al. (23) was applied, with minor modifications. All surgery was started and completed between 8:00 and 10:00.

Animals were anaesthetized with $4 \%$ isoflurane (Attane Vet, Piramal Healthcare, Northumberland, UK) delivered in pure oxygen (1 $1 / \mathrm{min})$ in an induction chamber until the righting reflex was absent. The anaesthetized animal was moved to a designated operation area, consisting of a polystyrene tray covered with an absorbent, disposable underpad, and placed in sternal recumbency. Anaesthesia was continuously delivered with $3 \% \pm 1$ isoflurane with an oxygen flow of $0.51 / \mathrm{min}$, and continuously adjusted to maintain relevant anaesthetic depth according to observed clinical parameters. Prior to surgery, anaesthetized animals were given eye ointment (Viscotears ${ }^{\circledR}$; Laboratoires Théa, Clermont-Ferrand, France) to prevent drying of the cornea, and injected subcutaneously with $0.5 \mathrm{ml}$ sterile saline as perioperative fluid therapy. The right hind paw was cleaned with iodine solution. Under aseptic conditions, a sterile transparent draping was placed by the surgeon to cover the entire animal, except for the right hind paw. The toes of the right hind paw were fixated using a purpose-made curved sterile needle and the paw was again swabbed with iodine solution. Using a scalpel with no. 10 blade, an incision was made, starting $0.5 \mathrm{~cm}$ from the edge of the heel and extending $1 \mathrm{~cm}$ in a longitudinal, distal direction in the plantar surface of the right hind paw, penetrating skin and 
Table I. Welfare assessment score sheet.

\begin{tabular}{|c|c|c|}
\hline Observation & & Score \\
\hline \multirow[t]{3}{*}{ General condition } & Awake and alert, normal response & 0 \\
\hline & Burrows or hides, but moves when touched or handled & 0.1 \\
\hline & Still and inactive, does not move, or reacts with fright or aggression reaction when touched or handled & 0.4 \\
\hline \multirow[t]{3}{*}{ Porphyrin staining } & None & 0 \\
\hline & Mild staining around eyes and nostrils & 0.1 \\
\hline & Obvious staining around eyes, face and paws & 0.4 \\
\hline \multirow[t]{3}{*}{ Gait and posture } & Normal & 0 \\
\hline & Mild incoordination when stimulated, hunched posture, mild piloerection & 0.1 \\
\hline & Obvious ataxia or head tilt, hunching, severe piloerection & 0.4 \\
\hline \multirow{3}{*}{$\begin{array}{l}\text { Body weight loss compared } \\
\text { to preoperative weight }\end{array}$} & $<5 \%$ & 0 \\
\hline & $5-10 \%$ & 0.1 \\
\hline & $10-20 \%$ & 0.4 \\
\hline \multirow[t]{3}{*}{ Appetite } & Normal food and water intake, signs of normal urination and defecation & 0 \\
\hline & No signs of food intake but drinks water and shows no signs of dehydration & 0.1 \\
\hline & No signs of food or water intake, signs of dehydration & 0.4 \\
\hline \multirow[t]{2}{*}{ Self-injury } & None & 0 \\
\hline & Bites or scratches itself, leading to wounds & 0.4 \\
\hline
\end{tabular}

Modified from the score sheet by Hampshire et al. (27).

fascia. The flexor muscle (m. plantar flexor digitorum brevis) was exposed and lifted from the surrounding tissue by blunt dissection, using a curved splinter forceps and small curved surgical scissors. Once elevated and fixed, the muscle layer was manipulated with 10 strokes with the non-cutting edge of the blade, in order to induce a minor injury to the muscle. Gentle hemostasis was applied and the incision was closed with three simple interrupted sutures using coated polyfilamentous suture material (Coated Vicryl ${ }^{\mathrm{TM}}$ 5-0. absorbable suture, reverse cutting needle; Ethicon, Somerville, NJ, USA) and cleaned using sterile saline.

Following surgery, the animals were placed in a dry, clean cage and kept under continuous surveillance until full consciousness was regained.

The experimenters had been thoroughly trained in the surgical procedure prior to the experimental series.

Electronic von Frey test. Postoperative pain was assessed by testing for mechanical hyperalgesia $(24,25)$, using the EVF algometer system, model BSBIOEVF3 with hard eppendorf tips (Bioseb, Vitrolles, France). Two baseline measurements were made on day -5 . Then the animals were tested twice on the day of surgery (day 0 ) at 1 and 6 h postoperatively) and once daily on day $1-3$, with day 3 being the day of euthanasia.

For testing, the animals were placed on an elevated metal grid floor with four individual, closed plastic cages $(16.5 \times 24 \times 14.5 \mathrm{~cm})$ (23). The animals were left to acclimatize for $30 \mathrm{~min}$ prior to each round of testing. To avoid bias from startling the animals, pretesting was performed with three non-recorded applications of the EVF tip with increasing force from underneath and through the holes of the metal grid floor on the left, non-injured hind paw (23). Then, five measurements were performed on the right, surgically treated hind paw, with approximately $10 \mathrm{~s}$ between each measurement. The EVF tip was applied perpendicularly to the skin, adjacent to the incision at the medial aspect of the paw, while avoiding direct contact with the incision. The EVF tip was only applied when the animals were standing in a natural position with complete weight bearing, meaning all four paws were to be securely on the grid and with equivalent pressure, when applying the EVF tip. The pain threshold was defined as the point of a pedal withdrawal response in the animal, with or without vocalization, where the EVF algometer automatically locked the withdrawal threshold in grams. The results were recorded by an observer who was not the same person as the operator of the algometer. All animals were tested by the same operator throughout the study.

The operators had been thoroughly trained in the EVF testing procedure prior to the experimental series.

Rat grimace scale. The RGS scoring was performed on all animals on day -5 , day 0 (day of surgery), and days $1-3$, with day 3 being the day of euthanasia. The animals were scored following an acclimatization period of approximately $30 \mathrm{~min}$ with the animals staying in their cages, although with the plastic hide removed to facilitate the observation of the animals. Two observers performed the scoring of the same animal on the same occasion in order to minimize variation.

The RGS scoring system used in this study consisted of evaluation of the four pain-related facial features ("action units") described by Sotocinal et al. (26) i) Orbital tightening; a narrowing of the orbital area resulting in partial or complete closure of the eye as an indicator of pain; ii) nose and cheek flattening; reduced bulging of the nose and cheek, reduction of the crease between the cheek and whisker pads, as indicators of pain; iii) ear changes; folding, curling and outward or forward angling of the ears, resulting in changes in the ear shape, and widening of the space between the ears, as indicators of pain; and iv) whisker change; backwards or outwards angling of the whiskers, bunching of the whiskers, as indicators of pain.

For each action unit, a score of 0,1 or 2 was assigned by the observer, with 0 representing the action being absent, 1 representing the action being moderately present and 2 representing the action being pronounced. The score for each action unit was set to the 

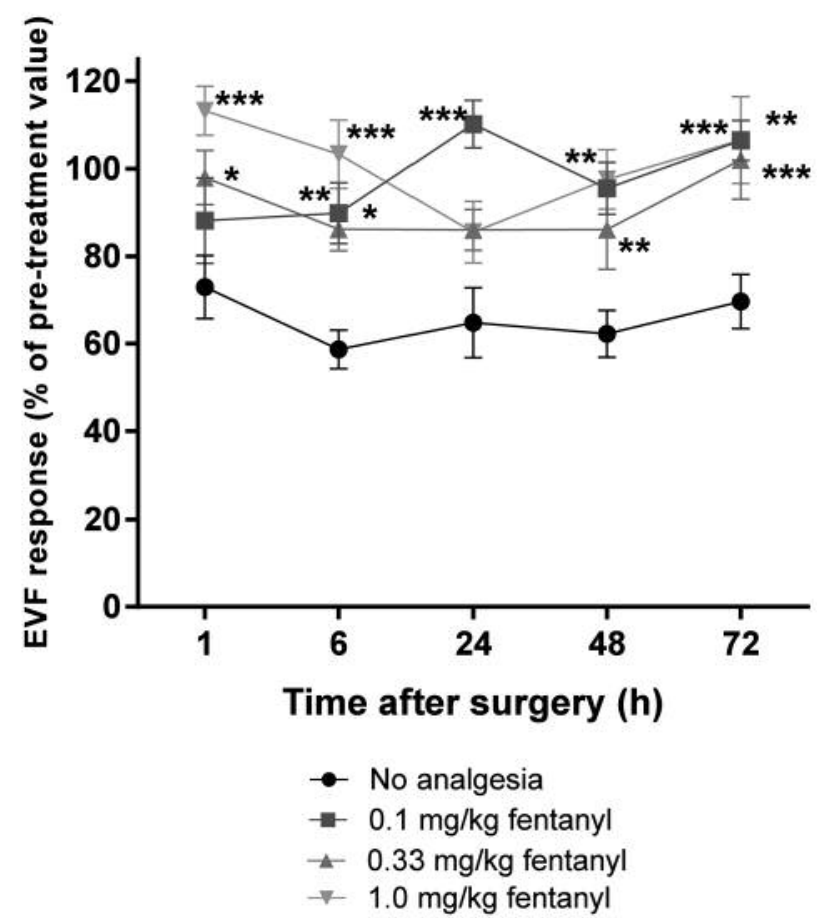

Figure 1. Response latency in the electronic von Frey test (EVF) after application of transdermal fentanyl, compared to non-treated control animals. Data are presented as the mean \pm standard error of the mean (SEM) percentage of preoperative values. Significantly different from the control group (no analgesia) at: $* p<0.05, * * p<0.01$ and $* * * p<0.001$, respectively, as determined with a two-way ANOVA with Bonferroni post-hoc tests.

average score of the two observers. The RGS score for each animal and day was the sum of the action unit scores. Thus, the maximal RGS score was 8 (in steps of 0.5 ).

Body weight changes. Body weight was recorded on days -5 , and days 0-3, between 9:00 and 13:00.

Welfare assessment. The overall well-being of the animals was assessed using a welfare monitoring scheme (Table I), based on the scoring scheme described by Hampshire et al. (27).

Welfare assessment was performed with the animals in their cages, with the plastic hide removed to facilitate the observation of the animals. Two observers performed the monitoring of the same animal on the same occasion in order to minimize variation. The welfare monitoring was performed on days -5 , and 0-3. If an animal was given a total welfare assessment score of 0.4 or higher, it was immediately euthanised for humane reasons.

Statistical procedures. All graphs and statistical calculations were made using GraphPad Prism version 7.00 (GraphPad Software Inc., La Jolla, CA, USA). Differences with $p$-values of less than 0.05 were considered statistically significant. The EVF response for each group was normalized with the value at day -5 as starting value, and the subsequent measurements are expressed is a percentage of the
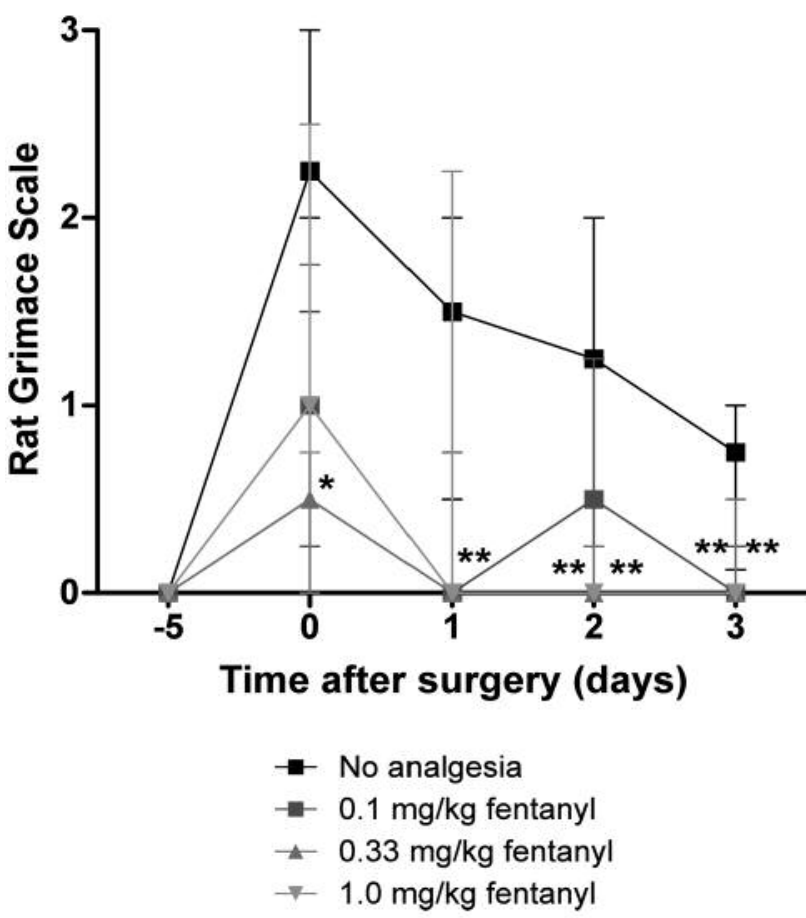

Figure 2. Pain-related facial expression, assessed according to the Rat grimace scale (RGS), after surgery and application of transdermal fentanyl, compared to non-treated control animals. Data are presented as the median \pm interquartile range. Significantly different from the control group (no analgesia) at: $* p<0.05$ and $* * p<0.01$, respectively, as determined with Kruskal-Wallis test with Dunn's multiple comparison test.

starting value. The different groups were compared with each other using a two-way repeated-measures analysis of variance (two-way (ANOVA), with time and treatments as variables, followed by Bonferroni post-hoc test. The RGS score is expressed as the median \pm interquartile range for each treatment group and day. The different groups were compared with each other using a KruskalWallis test, followed by Dunn's post-hoc test. Body weight is expressed as the mean \pm standard error of the mean for each treatment group and day. The different groups were compared with each other as per the EVF score. The welfare assessment score is expressed as the percentage of animals in each group and day that had any welfare implication registered (score $>0.1$ ). No statistical comparison was computed for the welfare assessment.

\section{Results}

Electronic von Frey test. Treatment with transdermal fentanyl, at all doses tested, significantly reduced nociceptive response in the EVF test throughout the $72 \mathrm{~h}$ of experimentation, as shown in Figure 1. The $0.1 \mathrm{mg} / \mathrm{kg}$ dose of fentanyl reduced the response at $6 \mathrm{~h}(p<0.01), 24 \mathrm{~h}(p<0.001), 48 \mathrm{~h}(p<0.01)$, and $72(p<0.001)$ after surgery; the $0.33 \mathrm{mg} / \mathrm{kg}$ dose reduced it at $1 \mathrm{~h}(p<0.05), 6(p<0.05)$, and $72 \mathrm{~h}(p<0.01)$; and the 1.0 


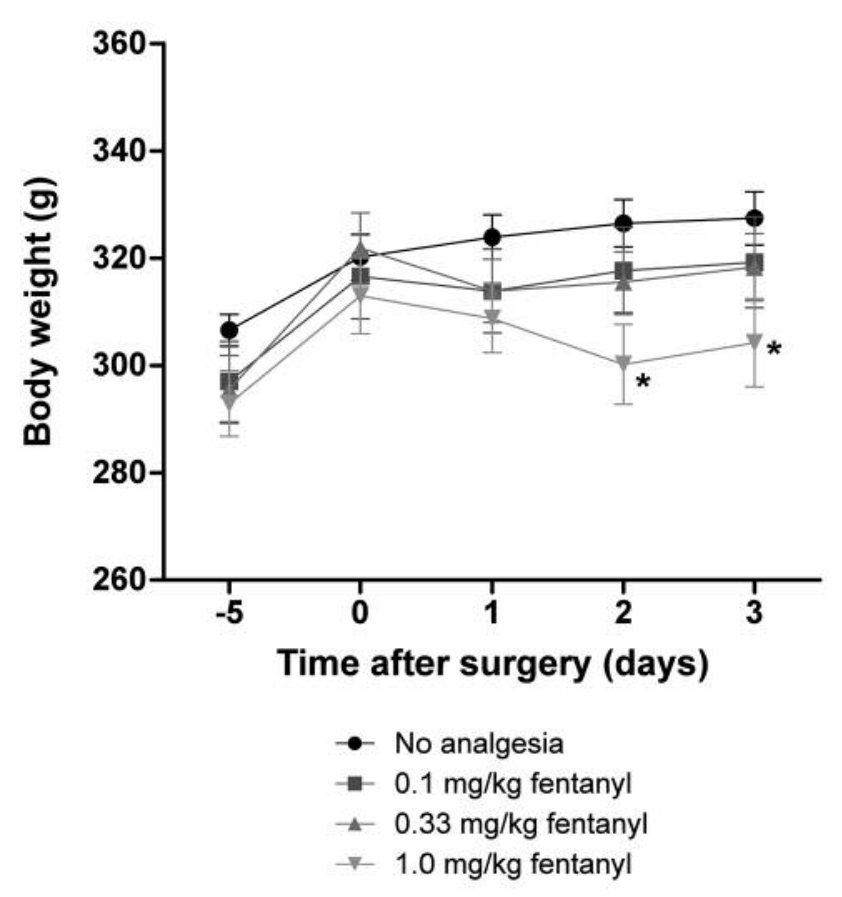

Figure 3. Daily body weight before and after surgery and application of transdermal fentanyl, compared to non-treated control animals. Data are presented as mean \pm standard error of the mean (SEM). *Significantly different from the control group (no analgesia) at $p<0.05$.

$\mathrm{mg} / \mathrm{kg}$ dose reduced it $1 \mathrm{~h}(p<0.001), 6 \mathrm{~h}(p<0.001), 48 \mathrm{~h}$ $(p<0.01)$, and $72 \mathrm{~h}(p<0.001)$.

Rat grimace scale. The RGS scoring demonstrated that the facial expression of the animals indicated no pain at day -5 , as shown in Figure 2. On the day of surgery (day 0), and during the remainder of the study, the RGS was above zero in the control group, with a median score ranging from 2.25 on day 0 to a score 0.75 on - day 3 . There was a significant difference between the fentanyl treated animals and the control animals from days 0 to 3 .

Body weight. The postoperative body weight was not affected either by the surgery or the fentanyl treatment, except for a reduced body weight gain compared to the control group during the last 2 days of the experiment after treatment with $1.0 \mathrm{mg} / \mathrm{kg}$ fentanyl (Figure 3).

Welfare assessment. The welfare assessment score is presented in Figure 4 as the incidence (as a percentage) of a welfare score above zero in the animals for each group. Due to the small sample size, it was not possible to compute any relevant statistics of incidence. However, the postoperative welfare scores showed a tendency towards improvement

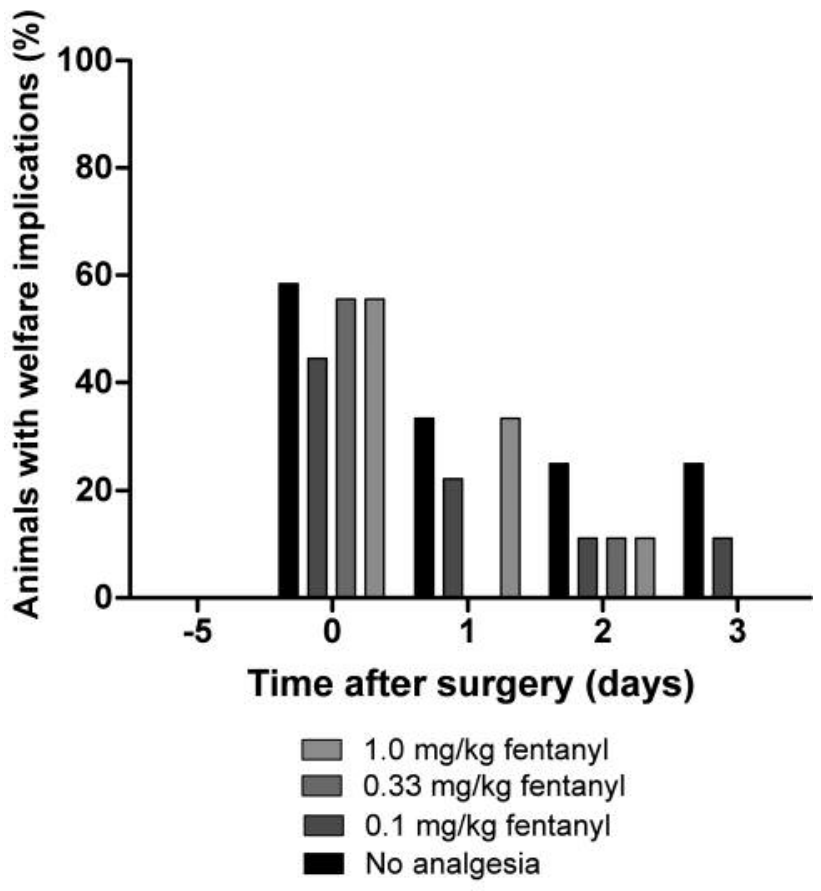

Figure 4. Descriptive data of the percentage of animals in each group that were found to have welfare implications in daily welfare monitoring.

after fentanyl treatment compared to non-treated animals on days 2 and 3 (Figure 4).

\section{Discussion}

The findings presented in this study demonstrate that the transdermal solution Recuvyra holds good potential for future treatment of postoperative pain. The compound showed analgesic effect in both the EVF test and in the RGS scoring, as hypothesized. The EVF test measures an induced response to tactile hyperalgesia $(24,25)$, which is directly related to the trauma and inflammatory reaction from the surgical incision and subsequent sound healing process. The RGS score, on the other hand, is considered a measurement of the adverse experience by the animal, and has been suggested to be an expression of the spontaneous pain experience (26). This is not considered the case with induced responses that reflect nociception rather than pain. Although both the EVF test and the RGS score have their limitations, they are both validated tests of analgesic effect from different perspectives, which strengthens the idea of using transdermal fentanyl solution for postoperative treatment of pain in rats.

It was hypothesized that fentanyl treatment would prevent body weight loss postoperatively, compared to non-treated 
animals. This turned out not to be the case. The surgical procedure caused neither any significant decrease in body weight in non-treated animals, nor any decrease in body weight gain compared to naïve non-operated animals (data not shown). This indicates that the surgical procedure was not severe enough to affect the appetite of the animals. On the other hand, it was observed that the body weight of the animals treated with the highest dose of fentanyl was lower compared to the non-treated animals on days 2 and 3. This is most likely due to the known appetite-reducing effects of opioids $(13,28)$, and might suggest that the highest dose applied in the present study was higher than necessary.

The welfare assessment scheme applied in the study was primarily used to identify humane endpoints at an early stage, and was thus not intended to form the basis for any statistical calculations. Nevertheless, it was observed that all groups had animals with some degree of welfare implications after surgery. Although none of the animals reached a score at the level necessitating euthanasia, the welfare monitoring indicated that the surgical procedure indeed inflicted welfare implications that called for analgesic treatment. Thus, this strengthens the validity of the procedure as a model for postoperative pain, and future application of transdermal fentanyl solution as a perioperative analgesic strategy should therefore be relevant.

Despite the promising findings of the present study, there are some concerns that need to be addressed. Application of Recuvyra in dogs has been successful but is not unproblematic. Studies have revealed that there may be variation in therapeutic as well as adverse effects between individual animals $(18,19)$. It is unlikely that the situation should be different in rats. Furthermore, the fact that the animals may groom each other after application calls for single housing, which is problematic when working with social animals such as rats. In the present study, the animals were single-housed throughout the entire study period. Such a long duration might not be necessary when applying the fentanyl solution in a different experimental setup, but some period of single housing cannot be avoided. Another study limitation that must be addressed is the fact that there were no control groups for animals undergoing anesthesia only without surgery or animals receiving fentanyl without surgery. We do not, however, think it is likely that such control groups would have altered the conclusions drawn from the present data. From a wider perspective, it would be of interest to know more about the effects of transdermal fentanyl on behavior in general. This was not the scope of the present study but should be investigated in future studies.

In conclusion, the transdermal fentanyl solution used in the present study appears to provide effective, non-invasive and long-lasting analgesia in laboratory rats. However, more studies need to be undertaken in order to verify the effect and to shed light on the concerns regarding varying serum concentrations and necessity for single-housing when using this postoperative regimen.

\section{Ethical Statement}

All experimental protocols involving animals were ethically reviewed and approved by the Danish Council for Animal Experiments. The protocols were also reviewed by the local animal welfare body at the University of Copenhagen.

\section{Conflicts of Interest}

The Authors declare no conflicts of interest in regard to this study.

\section{Acknowledgements}

This study was performed with support from the Copenhagen Center for Applied Animal Research (CALAR). The Authors also wish to thank Trine Marie Glahder and Helle Porsdal for technical assistance, and Dr. Carsten Grøndahl for introducing us to the transdermal fentanyl solution in the first place.

\section{References}

1 Goldkuhl R, Klockars A, Carlsson HE, Hau J and Abelson KS: Impact of surgical severity and analgesic treatment on plasma corticosterone in rats during surgery. Eur Surg Res 44: 117-123, 2010.

2 Morton DB and Hau J: Welfare assessment and humane endpoints. In: Handbook of Laboratory Animal Science: Volume 1-Essential Principles and Practices. Hau J and Schapiro SJ (eds.). Boca Raton: CRC Press, pp. 535-572, 2011.

3 Woodman DD: The adrenal glands. In: Laboratory Animal Endocrinology: Hormonal Action, Control Mechanism and Interactions with Drugs. Chichester: John Wiley and Sons Ltd., pp. 253-286, 1997.

4 Goldkuhl R, Carlsson HE, Hau J and Abelson KSP: Effect of subcutaneous injection and oral voluntary ingestion of buprenorphine on postoperative serum corticosterone levels in male rats. Eur Surg Res 41: 272-278, 2008.

5 Jessen L, Christensen S and Bjerrum OJ: The antinociceptive efficacy of buprenorphine administered through the drinking water of rats. Lab Anim 41: 185-196, 2007.

6 Flecknell PA, Roughan JV and Stewart R: Use of oral buprenorphine ('buprenorphine jello') for postoperative analgesia in rats-a clinical trial. LabAnim 33: 169-174, 1999.

7 Hovard A, Teilmann A, Hau J and Abelson K: The applicability of a gel delivery system for self-administration of buprenorphine to laboratory mice. Lab Anim 49: 40-45, 2015.

8 Seymour TL, Adams SC, Felt SA, Jampachaisri K, Yeomans DC and Pacharinsak C: Postoperative analgesia due to sustainedrelease buprenorphine, sustained-release meloxicam, and carprofen gel in a model of incisional pain in rats (Rattus norvegicus). J Am Assoc Lab Anim Sci 55: 300-305, 2016.

9 Abelson KSP, Jacobsen KR, Sundbom R, Kalliokoski O and Hau $\mathrm{J}$ : Voluntary ingestion of nut paste for administration of buprenorphine in rats and mice. Lab Anim 46: 349-351, 2012. 
10 Goldkuhl R, Hau J and Abelson KSP: Effects of voluntarily ingested buprenorphine on plasma corticosterone levels, body weight, water intake, and behaviour in permanently catheterised rats. In Vivo 24: 131-135, 2010.

11 Goldkuhl R, Jacobsen KR, Kalliokoski O, Hau J and Abelson KS: Plasma concentrations of corticosterone and buprenorphine in rats subjected to jugular vein catheterization. Lab Anim 44: 337-343, 2010.

12 Hestehave S, Munro G, Pedersen TB and Abelson KSP: Antinociceptive effects of voluntarily ingested buprenorphine in the hot-plate test in laboratory rats. Lab Anim 51: 264-272, 2017.

13 Kalliokoski O, Abelson KS, Koch J, Boschian A, Thormose SF, Fauerby N, Rasmussen RS, Johansen FF and Hau J: The effect of voluntarily ingested buprenorphine on rats subjected to surgically induced global cerebral ischaemia. In Vivo 24: 641646, 2010

14 Molina-Cimadevila MJ, Segura S, Merino C, Ruiz-Reig N, Andrés B and de Madaria E: Oral self-administration of buprenorphine in the diet for analgesia in mice. Lab Anim 48: 216-224, 2014.

15 Chum HH, Jampachairsri K, McKeon GP, Yeomans DC, Pacharinsak C and Felt SA: Antinociceptive effects of sustainedrelease buprenorphine in a model of incisional pain in rats (Rattus norvegicus). J Am Assoc Lab Anim Sci 53: 193-197, 2014.

16 Foley PL, Liang H and Crichlow AR: Evaluation of a sustainedrelease formulation of buprenorphine for analgesia in rats. J Am Assoc Lab Anim Sci 50: 198-204, 2011.

17 Dobromylskyj P, Flecknell PA, Lascelles BD, Pascoe PJ, Taylor $\mathrm{P}$ and Waterman-Pearson A: Management of postoperative and other acute pain. In: Pain management in animals. Flecknell PA and Waterman-Pearson A (eds.): W.B. Saunders, pp. 81-145, 2000.

18 Linton DD, Wilson MG, Newbound GC, Freise KJ and Clark TP: The effectiveness of a long-acting transdermal fentanyl solution compared to buprenorphine for the control of postoperative pain in dogs in a randomized, multicentered clinical study. J Vet Pharmacol Ther 35(Suppl 2): 53-64, 2012.

19 Martinez SA, Wilson MG, Linton DD, Newbound GC, Freise KJ, Lin TL and Clark TP: The safety and effectiveness of a longacting transdermal fentanyl solution compared with oxymorphone for the control of postoperative pain in dogs: a randomized, multicentered clinical study. J Vet Pharmacol Ther 37: 394-405, 2014.
20 Institute of Laboratory Animal Research: Guide for the Care and Use of Laboratory Animals. Washington DC: The National Academies Press, 2011.

21 Mähler M, Berard M, Feinstein R, Gallagher A, Illgen-Wilcke B, Pritchett-Corning $\mathrm{K}$ and Raspa M: FELASA recommendations for the health monitoring of mouse, rat, hamster, guinea pig and rabbit colonies in breeding and experimental units. Lab Anim 48: 178-192, 2014.

22 European Medicines Agency: Recuvyra, fentanyl; Annex 1Summary of product characteristics http://www.ema.europa.eu/ docs/en_GB/document_library/EPAR_-_Product_Information/ veterinary/002239/WC500116194.pdf. (2011, accessed 16 May 2017).

23 Brennan TJ, Vandermeulen EP and Gebhart GF: Characterization of a rat model of incisional pain. Pain 64: 493-501, 1996.

24 Abelson KSP and Roughan JV: Animal models in pain research. In: Handbook of Laboratory Animal Science, Vol. II: Animal Models. Hau J and Schapiro SJ (eds.). Boca Raton: CRC Press, pp. 123-147, 2011.

25 Ängeby Möller K, Johansson B and Berge O-G: Assessing mechanical allodynia in the rat paw with a new electronic algometer. J Neurosci Meth 84: 41-47, 1998.

26 Sotocinal SG, Sorge RE, Zaloum A, Tuttle AH, Martin LJ, Wieskopf JS, Mapplebeck JC, Wei P, Zhan S, Zhang S, McDougall JJ, King OD and Mogil JS: The Rat grimace scale: A partially automated method for quantifying pain in the laboratory rat via facial expressions. Molecular Pain 7: 55, 2011.

27 Hampshire VA, Davis JA, McNickle CA, Williams L and Eskildson H: Retrospective comparison of rat recovery weights using inhalation and injectable anaesthetics, nutritional and fluid supplementation for right unilateral neurosurgical lesioning. Lab Anim 35: 223-229, 2001.

28 Brennan MP, Sinusas AJ, Horvath TL, Collins JG and Harding MJ: Correlation between body weight changes and postoperative pain in rats treated with meloxicam or buprenorphine. Lab Anim 38: 87-93, 2009.
Received February 20, 2018

Revised March 23, 2018

Accepted March 28, 2018 